\title{
Controladores fuzzy adaptativos para la optimización de un sistema chiller
}

\author{
Moreno G. Francisco. E. ${ }^{1} \mid$ Bandarra F. Enio P ${ }^{2} \mid$ Becerra V. José A.
}

Recibido:

Diciembre 12 de 2010

Aceptado:

Mayo 20 de 2011
Doctor en Ingeniería Mecánica, femgarcia@ufps.edu.co, Grupo de Investigación en Automatización y Control GIAC, Universidad Francisco de Paula Santander, Avenida Gran Colombia No. 12E-96B Colsag. Cúcuta - Colombia.

${ }^{2}$ Doctor en Ingeniería Mecánica, Laboratorio de Energía y sistemas térmicos - LEST, Universidad Federal de Uberlândia, Av. João Naves de Ávila, 2121 - Campus Santa Monica, Bloco 1M, Uberlândia - Brasil.

${ }^{3}$ Magister en Ingeniería Electrónica, Grupo de Investigación en Automatización y Control - GIAC, Universidad

Francisco de Paula Santander, Avenida Gran Colombia No. 12E-96B Colsag. Cúcuta Colombia.

\section{Resumen}

Los sistemas chiller, son ciclos de compresión por vapor utilizados en aplicaciones industriales y residenciales, operados de forma clásica e inadecuada desde el punto de vista del consumo de energía. Este trabajo experimental desarrollado en el laboratorio de Energía y sistemas térmicos de la Universidad Federal de Uberlândia (UFU), investiga algunas ventajas en el uso del compresor a velocidad variable y válvula de expansión electrónica con el objetivo de reducir el consumo de energía y elevar el coeficiente de eficacia (COP) de estos sistemas chillers. En estas condiciones es propuesto un modelo experimental basado en técnicas de planeamiento experimental, aplicando herramientas de superficie de respuesta. Este modelo permite estimar el comportamiento dinámico de la frecuencia del compresor, para contribuir a lazos de control aplicando estrategias fuzzy adaptativas, presentando un excelente rendimiento en el sistema, buscando mínimos grados de supercalentamiento en el orden de los $7{ }^{\circ} \mathrm{C}$ y alcanzando mejores regiones de COP.

Palabras clave: Chiller, Control, Fuzzy, Refrigeración, Supercalentamiento, Superficie.

\section{Abstract}

The chiller systems are vapor compression cycles used in industrial and residential applications, operated a classical control and inadequate under energy consumption point of view. The experimental work, development in the Energy and Thermal System Laboratory of Federal University of Uberlandia (UFU), investigates some advantages of the use of variable speed compressor and electronic expansion of performance, (COP) of these chillers systems. In these conditions, a proposed model experimentally based on experimental planning techniques, applying tools of answer surfaces. This model allows the estimative of the dynamic behavior of the compressor frequency to contribute in the control mesh applying adaptative fuzzy strategies, showing a good performance on the system, searching a minimum degree of superheating in the order of the $7^{\circ} \mathrm{C}$ and reaching on the bests $\mathrm{COP}$ zones.

Keywords: Chiller, Control, Fuzzy, Refrigeration, Superheating, Surface. 


\section{Introducción}

$\mathrm{T}$ eóricamente el más eficiente método de gerenciamiento de ciclos de compresión por vapor, consiste en el control del grado de supercalentamiento del refrigerante a partir de la válvula de expansión, y en el manejo de la velocidad del compresor [1]. Recientes investigaciones han obtenido grandes mejorías en la eficiencia en este tipo de sistemas utilizando velocidad variable del compresor para garantizar el control de la temperatura [2-4] entre otros. La válvula de expansión termostática (VET) no puede corresponder a muchas necesidades de estos sistemas, por lo tanto, las válvulas de expansión electrónicas (EEV) comienzan a tener influencia en esta área, por ejemplo han sido utilizadas para cambiar la velocidad del refrigerante controlando la temperatura de evaporación y la velocidad del compresor [5].

La búsqueda por técnicas para el control de sistemas de refrigeración chiller y aire condicionado que buscan obtener mejores rendimientos térmicos y mecánicos han crecido en estos días actuales, con la consigna de proporcionar una efectiva integración de estos con la microelectrónica y computadores, lo que sumistra una amplia y continua posibilidad de nuevas soluciones. Este trabajo presenta la construcción, instrumentación, control y evaluación de un sistema experimental chiller de compresión por vapor; integrando los principios de proyecto térmico, proyecto electrónico, programación y técnicas de control fuzzy en el ajuste estricto del grado de supercalentamiento en función de las principales variables del proceso.

\section{Materiales y métodos}

\section{Banco de pruebas experimentales.}

El banco experimental fue proyectado para el levantamiento de los datos experimentales, y está compuesto por dos circuitos básicos: un principal de refrigerante HCFC (R22) y un secundario de agua, siendo este responsable por el enfriamiento del refrigerante en el circuito principal. La Figura 1, ilustra el banco experimental construido para los ensayos. La circulación del fluido refrigerante es generada por un compresor semi-hermético, modelo Octagon de la empresa Bitzer compresores. El intercambiador evaporador, con capacidad de $17.5 \mathrm{~kW}$ (5 TR), es de tipo "tube-in-tube", de tubos concéntricos, donde el refrigerante fluye en el interior del tubo central y el agua fluye en el espacio anular del tubo externo.

Para seguridad del compresor, fue instalado un separador de fase líquida después del evaporador, evitando posibles riesgos de entrada del refrigerante en fase líquida en el compresor. Se utiliza un intercambiador condensador de la misma capacidad del evaporador, también elaborado en tubos concéntricos. Los dos intercambiadores de calor fueron aislados térmicamente, evitando al máximo la perdida de calor hacia el medio ambiente. Para el enfriamiento del agua de condensación, fue utilizado un circuito secundario compuesto por una torre de enfriamiento, no mostrada en el esquema del banco de ensayos. El agua de alimentación del intercambiador evaporador, responsable por la variación de la carga térmica en el sistema, proviene de un tanque aislado que contiene una resistencia eléctrica, con potencia de $15 \mathrm{~kW}$, para controlar la temperatura del agua en la entrada del intercambiador evaporador en valores previamente estipulados en los ensayos.

Figura 1. Esquema del banco de ensayos.

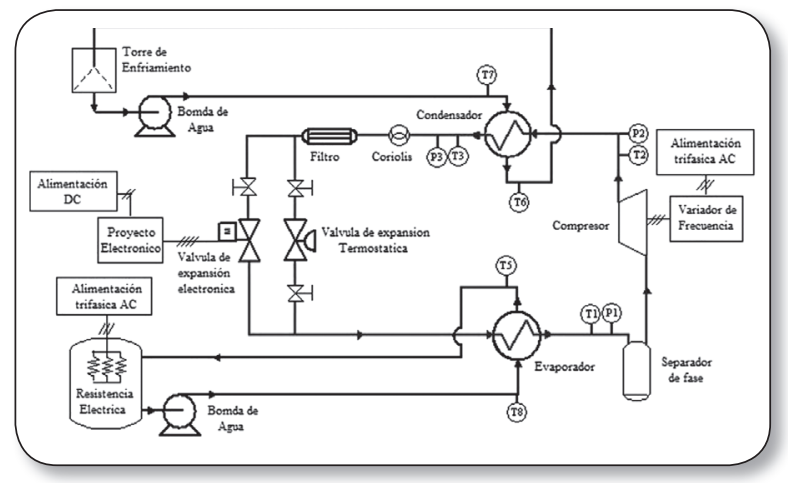


En relación a la instrumentación, para las medidas de temperatura fueron utilizados sensores de tipo PT100 y para presión, sensores do tipo piezo-resistivos. Fue utilizado, un variador de frecuencia para ajustar la velocidad de rotación del compresor. Un medidor de flujo másico tipo coriolis fue adicionado al circuito principal, complementando la instrumentación. La adquisición de dados fue realizada por circuitos de acondicionamiento de señal con salida analógica $\pm 10 v d c$. La conversión analógica/digital se llevo acabo usando un controlador lógico programable (PLC), y los datos fueron monitoreados y controlados a través de un software desarrollado en LABVIEW. El sistema cuenta, además, con una válvula de expansión termostática (TXV) de 5 TR, paralelamente en bypass, como muestra la figura 1 , fue instalada una válvula de expansión electrónica (EEV) marca Danfoss, modelo ETS25, con motor de paso-paso bipolar operado electrónicamente. La precisión de los parámetros calculados, fue determinada de acuerdo al procedimiento sugerido por [6], con intervalo de confianza del $95 \%$, para las medidas de temperatura con una incertidumbre $\pm 0.15 \mathrm{~K}$, para la presión $\pm 0.30 \%$, para el flujo másico $\pm 0.15 \%$, y para la potencia consumida $\pm 0.037 \mathrm{~kW}$.

\section{Obtención del modelo para un sistema chiller}

Con el objetivo de obtener un conocimiento del comportamiento estático de las variables, el sistema chiller de refrigeración fue sometido a un planeamiento experimental DOE (Design of Experiments), a partir de condiciones en régimen permanente, a fin de analizar las tendencias de los parámetros del sistema en superficies de repuesta. Fue aplicado un arreglo central compuesto de cara centrada (CCF), estableciéndose dos factores actuantes en el sistema: número de pasos de la válvula de expansión (VEE) y frecuencia de rotación del compresor (FREQ) siendo las variables de estudio definidas en un valor máximo $(+1)$ y un valor mínimo $(-1)$, denominados niveles. La combinación de las respuestas de los ensayos permite la obtención de una correlación multi-variable (modelo lineal o cuadrático), que permite la compresión y la visualización del comportamiento de las variables del proceso en régimen permanente de la temperatura de evaporación (Tev), flujo másico del refrigerantes R22 $\left(\dot{m}_{R 22}\right)$ y el coeficiente de desempeño (COP), entre otros. Por lo tanto, la finalidad de este modelo es proporcionar salidas futuras en el sistema, predictivas para cada instante de tiempo y facilitar la ejecución de futuras acciones guiadas para llevar el sistema más próximo posible de una referencia estable. La figura 2, muestra la estructura experimental empleando el modelo propuesto experimentalmente.

Figura 2. Estructura Experimental: Lazo cerrado trabajando con el modelo propuesto experimental.

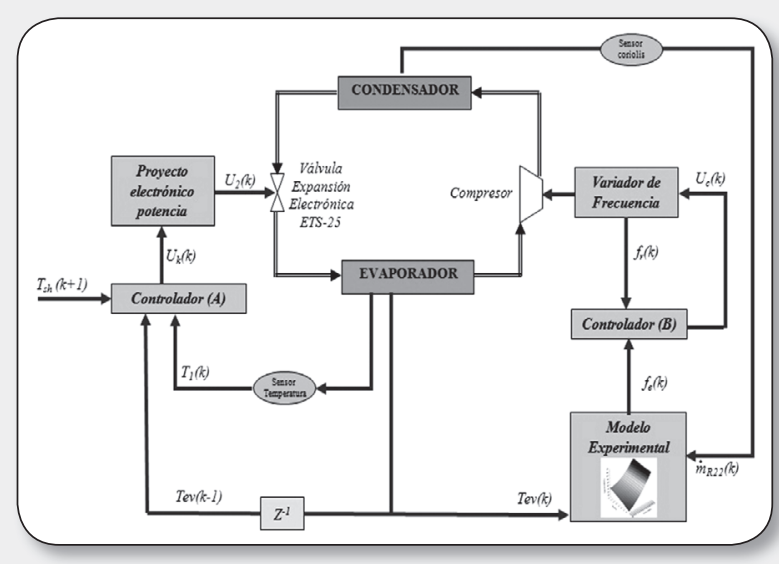

Implementación de los controladores fuzzy para el sistema chiller.

\section{A. Control del grado del supercalentamiento a través de la válvula de expansión.}

Elcontrolador denominado (A), comose puede observar en la figura 2, está fundamentado en la arquitectura de un controlador fuzzy convencional proporcional e integrativo (PI) desarrollado en este trabajo para controlar el motor de pasos de la válvula ETS25. Este controlador propuesto, como puede ser observado en la figura 3 , es de tipo sintonizado heurísticamente, admite como entrada: el error (e) y el error acumulado (ea) 
Respuestas

Año 16

No. 1

Junio 2011

ISSN 0122-820X en relación a la temperatura de evaporación con una señal de referencia. El resultado de este controlador fuzzy PI es una señal de salida $\left(U_{k}\right)$ codificado a tres bits, formato binario, multiplicado por una ganancia $\left(k_{p c}\right)$ igual a 1 introducida en el código maestro del controlador. Que finalmente genera una señal de salida para el actuador $\left(U_{2}\right)$ representando el numero de pasos para el cual el motor de la válvula deberá actuar.

Figura 3. Controlador fuzzy (A) propuesto para el grado del supercalentamiento a través de la válvula de expansión.

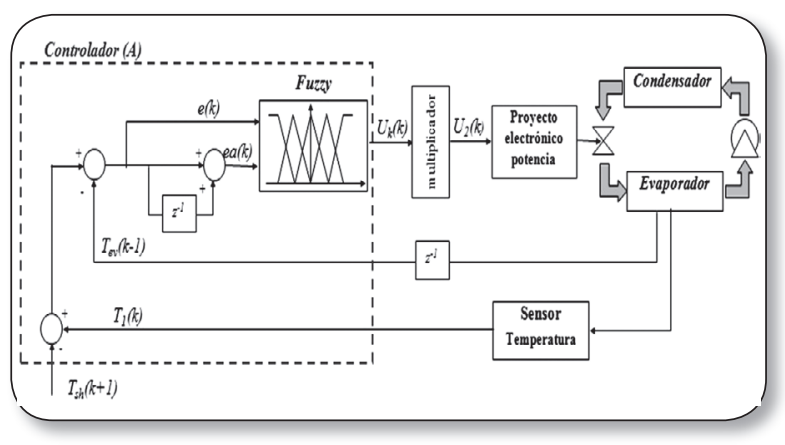

\section{B. Control de la frecuencia de rotación del} motor en el compresor.

Para mejorar la eficiencia del sistema chiller de compresión por vapor, se implementó otro control denominado (B), observado en las figura 3 y 4 , basado en una lógica difusa adaptativa para controlar la frecuencia de rotación del compresor. La relevancia de este tipo de controlador difiere de los controladores fuzzy convencionales, por poseer reajuste automático para adaptarse a nuevas características del proceso que se desea controlar [7].

La necesidad de identificar estos cambios y de adaptarse a nuevas condiciones, adiciona a los controladores fuzzy adaptativos dos componentes extras, que no están presentes en los controladores fuzzy convencionales. El primer componente es el "monitor del proceso" cuya función es detectar los cambios en las características del proceso permitiendo ser una medida del rendimiento de actuación del controlador o un parámetro basado en el estado del proceso. El segundo componente adicional es el "Mecanismo de adaptación", que a partir de la información proporcionada por el monitor del proceso actualiza los parámetros del controlador, tales como: factor de escala (ganancia) de cada variable, conjunto difuso de cada variable lingüística o las reglas de la base del conocimiento.

Figura 4. Controlador B difuso adaptativo propuesto para el control de la frecuencia del compresor.

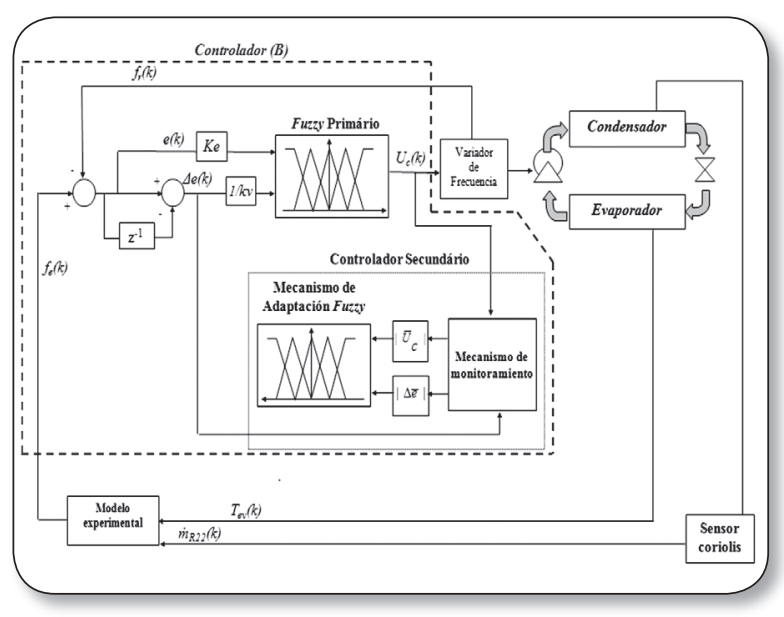

Así mismo para realizar el control de la frecuencia de rotación del compresor con base en la información anteriormente presentada (figura 4) fue realizado, inicialmente un controlador fuzzy primario y en seguida fue implementado un mecanismo fuzzy de adaptación de ganancia, donde sus variables de entrada del controlador fuzzy son evaluadas a partir de la señal del error que representa la diferencia entre la frecuencia estimada por el modelo propuesto $\left(f_{e}\right)$ y la frecuencia real del compresor $\left(f_{r}\right)$.

Para el mecanismo de adaptación, fue establecido un universo de discurso normalizado en un intervalo de -1 a 1 , acoplado al controlador fuzzy primario. Cabe destacar que el sistema es monitoreado a partir de la media absoluta de la derivada del error $(\Delta \bar{e})$ y de la media absoluta de la salida de control $\left(\bar{U}_{c}\right)$ observando los tres últimos tiempos de muestreo. 
De forma similar, fueron definidas para cada una de las entradas del mecanismo fuzzy de adaptación, tres funciones de pertinencia $[(\mathrm{P})$ pequeño, (M)medio y (G)grande] de tipo triangular, igualmente espaciadas definidas dentro del universo de discurso de las variables de entrada y sintonizadas de forma heurística. El mecanismo de inferencia aplicado fue el max-prod y la salida de tipo Sugeno de orden cero, asumiendo los valores $\mathrm{D}=0.8 ; \mathrm{M}=1 \mathrm{y}$ $\mathrm{A}=1.2$.

\section{Resultados y discusión}

Esta etapa tiene como objetivo la verificación de la respuesta de control sobre condiciones pré-establecidas, operando con el modelo propuesto experimentalmente obtenido. El sistema es puesto en funcionamiento con su controlador, operando en lazo cerrado, es monitoreado hasta alcanzar el régimen permanente, regulando simultáneamente, la abertura de la válvula de expansión y la frecuencia de rotación del compresor, además de mantener un grado de supercalentamiento establecido.

Para iniciar los ensayos, el sistema chiller de refrigeración es accionado hasta alcanzar la frecuencia nominal de rotación $(60 \mathrm{~Hz}$ equivalente a $1750 \mathrm{rpm}$ ). En la secuencia, es aplicado un cambio en la posición de la válvula de expansión, esperando que el sistema se estabilice nuevamente. En el primer ensayo realizado, la válvula fue cerrada hasta alcanzar una temperatura de evaporación (Tev) igual $-11.8^{\circ} \mathrm{C}$, con un grado de supercalentamiento $(T s h)$ igual a $27^{\circ} \mathrm{C}$, como se observa en la Figura 5. La carga térmica es proporcionada por la temperatura del agua en la entrada del intercambiador de calor evaporador $\left(T_{8}\right)$, manteniendo esa temperatura en el orden de $24^{\circ} \mathrm{C}$. Los flujos másicos de agua a la entrada del evaporador y condensador fueron establecidos en $0.23 \mathrm{~kg} / \mathrm{s}$ y $0.989 \mathrm{~kg} / \mathrm{s}$, respectivamente.

Después de un tiempo razonable (17 segundos en este ensayo), con el sistema operando en régimen estable, fue accionado el controlador, que actúo llevando el sistema a un nuevo régimen estable, siendo observado un tiempo de acomodación $t_{a}$ para estabilizarse de 218 segundos en este ensayo y un tiempo muerto observado de 15 segundos, temperatura de evaporación que en este ensayo no fue fijada y grado de subenfriamiento ( $T s c$ ) por encima de los $0^{\circ} \mathrm{C}$. Fue observado el control del grado de supercalentamiento que fue fijado en los diferentes ensayos en $7^{\circ} \mathrm{Co} 2^{\circ} \mathrm{C}$ y la evolución del COP.

En este primer ensayo, la tasa de transferencia de calor en el intercambiador de calor evaporador, o capacidad de enfriamiento, $\left(\dot{Q}_{e v}\right)$, registró un máximo valor de $\dot{Q}_{e v}$ del orden de $6.42 \mathrm{~kW}$ con potencia consumida de 2.1 $\mathrm{kW}$. El controlador mejoró el coeficiente de

Figura 5. Comportamiento de las temperaturas de evaporación y grado supercalentamiento para el primer ensayo experimental.

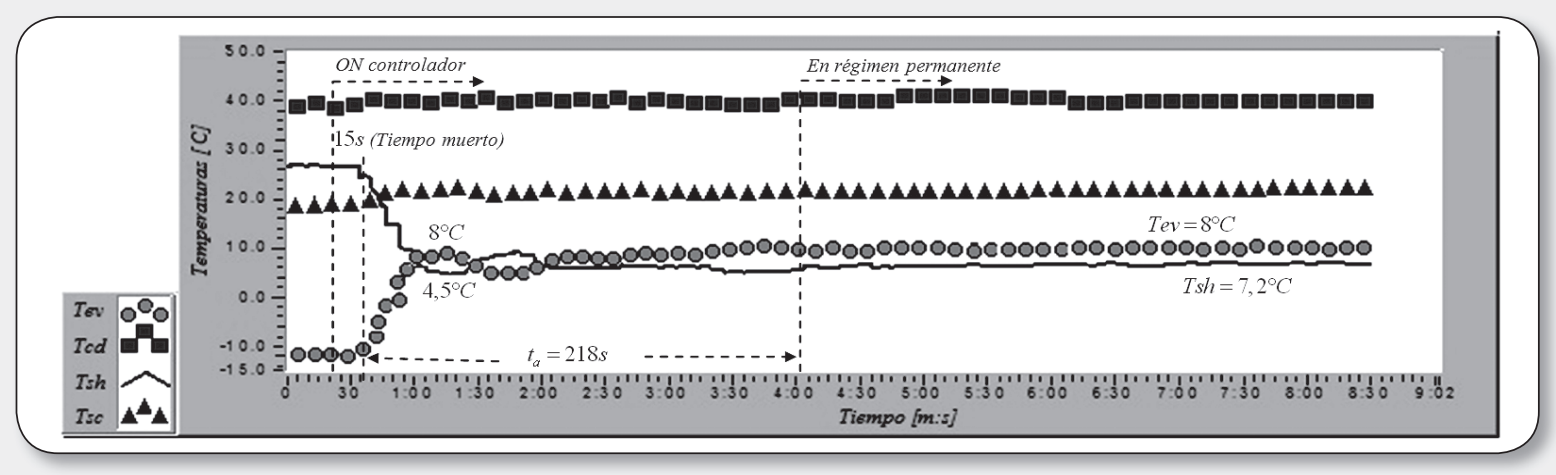


Respuestas

Año 16

No. 1

Junio 2011

ISSN 0122-820X rendimiento del sistema durante su actuación, presentando una mejoría en su valor, inicialmente de 1.5 , hasta 2.5 en régimen estable final.

Cuando el controlador es accionado, es detectada una diferencia entre la frecuencia estimada por el modelo y la frecuencia real, como puede ser observado en la figura 6a. El valor inicial de $k v$ como factor de adaptación del controlador observado en la figura $6 \mathrm{~b}$ es muy pequeño, lo que implica que la derivada del error tiene poca importancia para el controlador principal en los primeros instantes, envolviendo un número estricto de reglas. Por lo tanto, durante 26 segundos el controlador actúa sin ninguna compensación de la señal de salida $\left(U_{c}\right)$ para disminuir la frecuencia del compresor (trabaja tipo on-off).

Después de los 40 segundos, como es ilustrado en la figura $6 \mathrm{~b}$, el valor de $k v$, como mecanismo interno de adaptación, va aumentando y hace que el controlador se torne más sensible al $\Delta \mathrm{e}$, consecuentemente, un número mayor de reglas del controlador principal es utilizado, lo que mejora el desempeño del controlador. El valor inicial de $k v$ influencia, por lo tanto, en la aplicación del número de reglas en el controlador principal al inicializarse el proceso de control y la "velocidad" con que otras reglas son "alcanzadas". Así mismo, el controlador actúa generando salidas moduladas de manera que disminuya el error.

El valor de $k v$ fue monitoreado, paso a paso, por el mismo controlador adaptativo y después de 250 segundos, fue restablecido el valor del inicial, conforme la permanencia dentro de un rango de $\pm 1 \%$ por más de 20 instantes de muestreo. Como puede ser observado en la figura $6 \mathrm{~b}$, el valor de $1 / \mathrm{kv}$ convergió para un valor de $1 / 25$.

Fueron realizados posteriormente ocho ensayos similares, partiendo de un estado permanente

Figura 6. Efectos del controlador lógico fuzzy adaptativo en el primer ensayo experimental. (a) Evolución de las frecuencias de referencia y real del compresor. (b) Sensibilidad $(1 / k v)$ y señal de salida $\left(U_{c}\right)$ del controlador (B) fuzzy adaptativo.

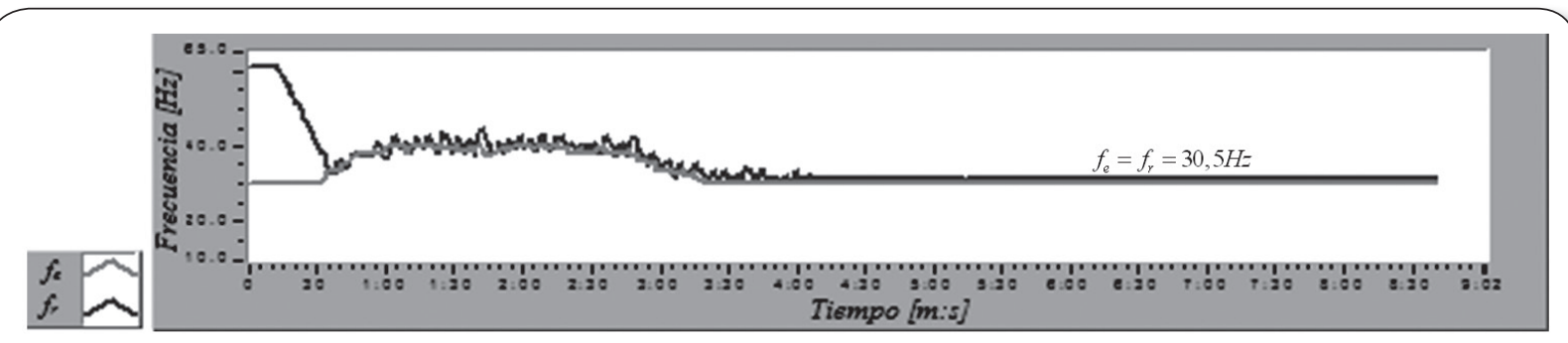

(a)

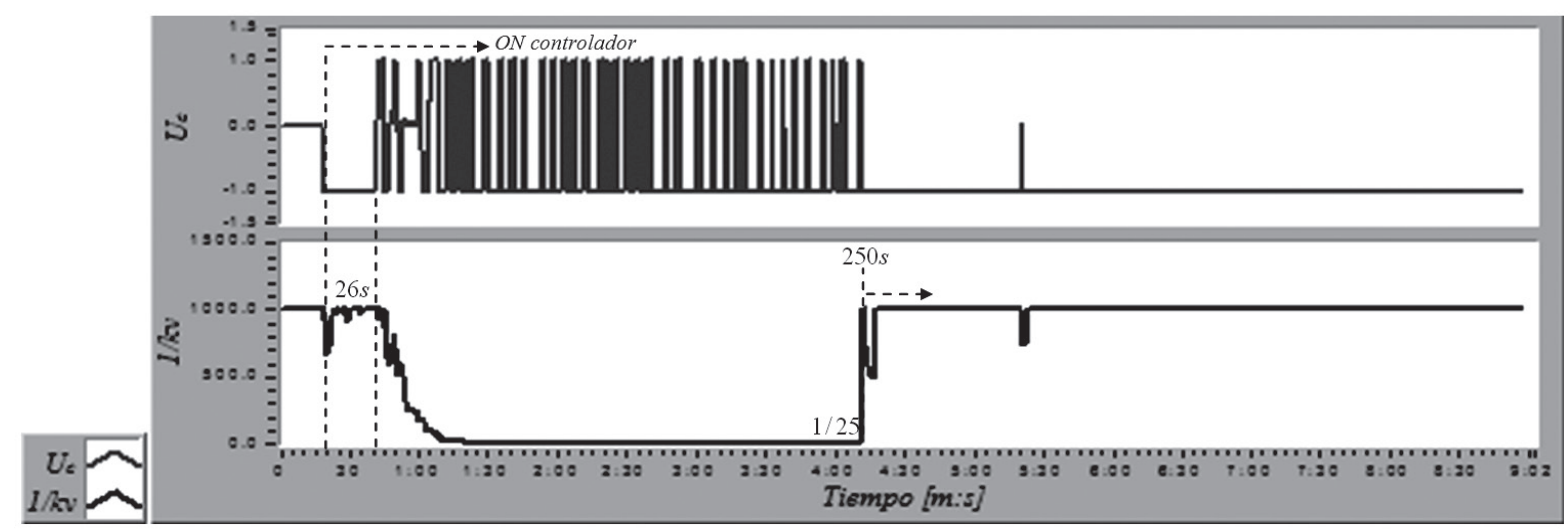

(b) 
inicial del sistema predeterminado, cambiando el grado de supercalentamiento deseado para $7^{\circ} \mathrm{C}$ o $2^{\circ} \mathrm{C}$, e iniciando a partir de frecuencias de rotación del compresor de $60 \mathrm{~Hz}$ y $35 \mathrm{~Hz}$, utilizando invariablemente el modelo del sistema obtenido experimentalmente.

Con el objetivo de sintetizar y comprender mejor el comportamiento y el efecto del controlador híbrido general, en la búsqueda del estado final deseado, la figura 7 presenta el COP, en función de la capacidad de enfriamiento, $\left(\dot{Q}_{e v}\right)$ y la temperatura de evaporación (Tev), para el primer y tercer ensayo, que trabajaron con la misma condición inicial (frecuencia inicial de rotación del compresor de $60 \mathrm{~Hz}$ ) y grado de supercalentamiento iniciales de $23^{\circ} \mathrm{C}$ y $27,1^{\circ} \mathrm{C}$ respectivamente. Se observa la trayectoria para el tercer ensayo (línea trazada) en el control del mínimo grado de supercalentamiento $\left(2^{\circ} \mathrm{C}\right)$, siguiendo a través de regiones de valor elevado del COP sin mayores oscilaciones, el cual es debido a la influencia del control simultaneo a la válvula de expansión y el compresor, lo que representa un total manejo de la dinámica del sistema.

Figura 7. Trayectoria del comportamiento del control, a través de superficies del coeficiente de rendimiento (COP), en función de la capacidad de refrigeración $\left(\dot{Q}_{e v}\right)$ y la temperatura de evaporación

$(T e v)$ para el primer y tercer ensayo partiendo de frecuencia inicial de $60 \mathrm{hz}$.

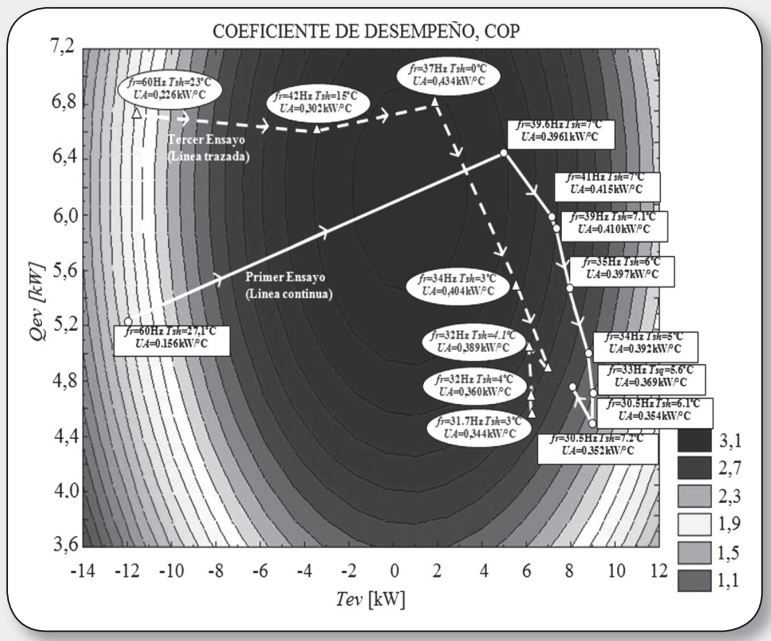

Un estudio comparativo entre los valores medios del COP obtenidos con diferentes estrategias de control utilizadas en este trabajo, son presentados en la figura 8 . Se observa la viabilidad que resulta al implementar controladores fuzzy adaptativos, representando un mejoramiento del COP medio por encima de 2 frente a la técnica clásica de control on-off.

Figura 8. Comparación de los diferentes rendimientos obtenidos con las diferentes estrategias de control usadas en sistemas de refrigeración de mediana capacidad.

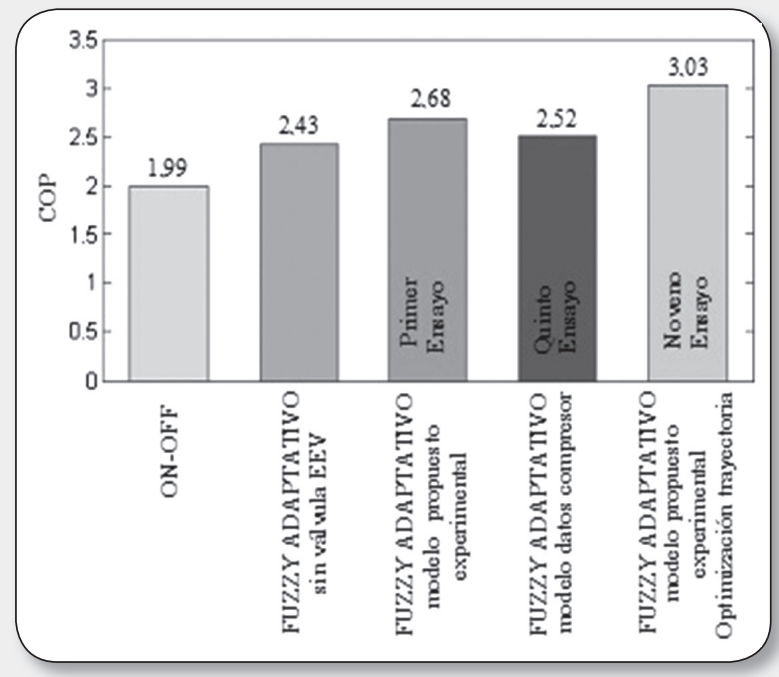

\section{Conclusiones}

La aplicación de herramientas, relativas al planeamiento de experimentos, proporciono un análisis de resultados de manera objetiva. El delineamiento experimental fue tratado de la forma más simple posible, de manera que reconozca la dinámica del sistema como un proceso interactivo de variables.

Fue desarrollado un modelo para el control del grado de supercalentamiento controlando simultáneamente la abertura de la válvula de expansión electrónica ETS25 y la frecuencia de rotación del compresor, aprovechando las ventajas ofrecidas por los controladores fuzzy.

La estrategia de control garantizó buena estabilidad al sistema. Se observó un óptimo desempeño del controlador en mantener el 
mínimo grado de supercalentamiento del refrigerante R22 en un nivel deseado y con un error mínimo aceptable $\pm 1^{\circ} \mathrm{C}$.

La importancia de estrategias de control, permitió ajustar el grado de supercalentamiento recorriendo directamente regiones de máximo $\mathrm{COP}$, experimentando siempre el mejor camino en la temperatura de evaporación y maximizando el coeficiente global de transferencia de calor en el orden de $0.40 \mathrm{~kW} /{ }^{\circ} \mathrm{C}$ a bajas frecuencias de rotación del compresor entre $35 \mathrm{~Hz}$ y $40 \mathrm{~Hz}$, siendo estas condiciones de trabajo optimas para este diseño experimental.

Finalmente, el hecho de trabajar con variador de frecuencia para este tipo de sistema, permitió una economía de energía eléctrica anual, además de la reducción de costos y mayor confiabilidad en relación al uso del sistema, favoreciendo el retorno de la inversión en un corto espacio de tiempo.

\section{Agradecimientos}

Los autores gratamente reconocen el suporte dado por la CNPq (Concejo Nacional de Desarrollo Científico y Tecnológico), CAPES y FAPEMIG. Los autores extienden este reconocimiento a la Bitzer BRASIL y ACP Termotecnica, por el compresor e intercambiador de calor donados para esta investigación.

\section{2}

\section{Referencias}

\section{Bibliográficas}

[1] Yasuda H. "Modeling of refrigeration cycle dynamics for air conditioner." transaction of jsme 1994;60(573):10310.

$\begin{array}{llr}\text { [2] } \begin{array}{l}\text { Shunagquan } \\ \text { representation }\end{array} & \text { S. } & \text { performance } \\ \text { variable-speed }\end{array}$ compressor for inverter air condidioners based on experimental data, int. j. refrigeration, vol 27, 2004, pp. 805- 815 .

[3] Koury R. N., Machado L.E, Ismail K. A. R. Numerical simulation of a variable speed refrigeration, int. j. refrigeration, vol. 24, 2001, pp. 192-200.

[4] Vargas J. V.C., Parise J. A.R. "simulation in transient regime of a heat pumb with closed-loop and on-off control", int. j. refrigeration, vol. 18, no. 4, pp. 235-243, 1995.

[5] Outtagarts A, Haberschill P, Lallemand M. Comportement dynamique d'un

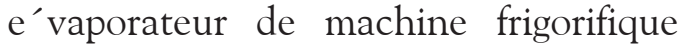
soumis a' des variations de debit. in: proceedings of $19^{\text {th }}$ international congress of refrigeration, b2. la Haya, Netherlands, 1995. p. 413-420.

[6] Albernethy R.B, Thompson J.W, Uncertainties in gas turbine measurements, isa i-483-3, 1980.

[7] Garcia F.E.M. "Estudo experimental de um controle adaptativo para um sistema de refrigeração." 2006. 108 f. tese de mestrado-universidade federal de Uberlândia, $M G$ - Brasil. 